IJMMS 32:1 (2002) 29-40

PII. S0161171202111136

http://ijmms.hindawi.com

(c) Hindawi Publishing Corp.

\title{
A CLASS OF GAP SERIES WITH SMALL GROWTH IN THE UNIT DISC
}

\section{R. SONS and ZHUAN YE}

Received 14 November 2001

Let $\beta>0$ and let $\alpha$ be an integer which is at least 2. If $f$ is an analytic function in the unit $\operatorname{disc} D$ which has power series representation $f(z)=\sum_{k=0}^{\infty} a_{k} z^{k^{\alpha}}, \limsup _{k \rightarrow \infty}\left(\log ^{+}\left|a_{k}\right| /\right.$ $\log k)=\alpha(1+\beta)$, then the first author has proved that $f$ is unbounded in every sector $\{z \in D: \phi-\epsilon<\arg z<\phi+\epsilon$, for $\epsilon>0\}$. A natural conjecture concerning these functions is that $\limsup _{r \rightarrow 1^{-}}(\log L(r) / \log M(r))>0$, where $L(r)$ is the minimum of $|f(z)|$ on $|z|=r$ and $M(r)$ is the maximum of $|f(z)|$ on $|z|=r$. In this paper, investigations concerning this conjecture are discussed. For example, we prove that $\limsup _{r \rightarrow 1^{-}}(\log L(r) / \log M(r))=1$ and $\limsup \operatorname{sul}_{r \rightarrow 1^{-}}(L(r) / M(r))=0$ when $a_{k}=k^{\alpha(1+\beta)}$.

2000 Mathematics Subject Classification: 30B30, 30D35.

1. Introduction. Let

$$
F(z)=\sum_{k=1}^{\infty} a_{k} z^{\lambda_{k}}
$$

represent an analytic function in the unit disc, and set

$$
\begin{aligned}
M(r) & =\max _{|z|=r}|F(z)|, \\
M\left(r ; \theta_{1}, \theta_{2}\right) & =\max _{|z|=r, \theta_{1} \leq \arg z \leq \theta_{2}}|F(z)|, \\
L(r) & =\min _{|z|=r}|F(z)| .
\end{aligned}
$$

Define

$$
\begin{gathered}
M_{2}(r)=\left(\frac{1}{2 \pi} \int_{0}^{2 \pi}\left|f\left(r e^{i \theta}\right)\right|^{2} d \theta\right)^{1 / 2}, \\
M_{2}(r ; \phi-\delta, \phi+\delta)=\left(\frac{1}{2 \delta} \int_{\phi-\delta}^{\phi+\delta}\left|f\left(r e^{i \theta}\right)\right| d \theta\right)^{1 / 2} .
\end{gathered}
$$

The growth order $\rho$ of the function $F$ is defined by

$$
\rho=\limsup _{k \rightarrow \infty} \frac{\log ^{+} \log ^{+}\left|a_{k}\right|}{\log \lambda_{k}-\log ^{+} \log ^{+}\left|a_{k}\right|},
$$

or

$$
\rho=\limsup _{r \rightarrow 1^{-}} \frac{\log \log M(r)}{-\log (1-r)}
$$


In 1916, Wiman [6] proved that

$$
\limsup _{r \rightarrow 1^{-}} \frac{L(r)}{M(r)}=1
$$

if $F$ is an analytic function in unit disc and satisfying

$$
\liminf _{k \rightarrow \infty} \frac{\log \left(\lambda_{k+1}-\lambda_{k}\right)}{\log \lambda_{k}}>\frac{1}{2} \frac{2+\rho}{1+\rho}
$$

where $\rho$ is the order of $F$.

In 1975, Nicholls and Sons [3] constructed an analytic function in the unit disc which shows the above Wiman's result is best possible if the order $\rho>0$. They also proved, under some restriction on $\left\{\lambda_{k}\right\}$, that an analytic function of order $\rho(>0)$ takes every complex number infinitely many times in the unit disc. In 1989, Sons [5] studied analytic functions of order zero which have the power series representation

$$
f(z)=\sum_{k=1}^{\infty} a_{k} z^{k^{\alpha}}
$$

where $\alpha(>2)$ is an integer, $\beta$ is a positive real number, and

$$
\limsup _{r \rightarrow 1^{-1}} \frac{\log ^{+}\left|a_{k}\right|}{\log k}=\alpha(1+\beta) .
$$

Sons [5] proved the function $f$ is unbounded in any small sector of the circle centered at zero.

Since the theta function given by the series

$$
1+\sum_{n=1}^{\infty} 2 z^{n^{2}}=\prod_{n=1}^{\infty}\left(1+z^{2 n-1}\right)^{2}\left(1-z^{2 n}\right)
$$

is of order zero and never takes the value zero in the unit disc, Sons [5] asked whether the functions in (1.8) assume every complex number in the unit disc.

In this paper, we are continuing to study the gap series of the form (1.8), which is the only well-known gap series with the growth order zero. We first show that, for any $\delta>0$ and any $\phi \in[0,2 \pi), M(r ; \phi-\delta, \phi+\delta)$ is greater than $M_{2}(r)$ multiplied by an absolute constant on a sequence of $r$, which complements the results in [5]. Then we take a close look at the class of the gap series

$$
g(z)=\sum_{k=1}^{\infty} k^{\alpha(1+\beta)} z^{k^{\alpha}},
$$

where $\alpha(>2)$ is an integer, $\beta$ is a positive real number. The class (1.11) is taken as a good representative of the class (1.8). We show that the limit of the ratio of the minimum modulus to the maximum modulus of $g$ is zero. However, the limit superior of the ratio of the logarithmic minimum modulus to the logarithmic maximum modulus is one. Therefore, any function in (1.11) assumes every value in the complex plane, although the function grows very slowly. 
Moreover, the functions in (1.11) shed some light on the sharpness of the restriction (1.7) when $\alpha$ is very large since the left-hand side of $(1.7)$ is $(\alpha-1) / \alpha$ and the righthand side of that is 1 . We conjecture that the above Wiman's result is sharp. A detailed discussion of this matter can be found in [1] for entire function and in [2, 4] for analytic functions in the unit disc.

In what follows, we use the standard notation of function theory. We also denote the maximum term of $f$ on $|z|=r$ by $\mu(r)$, and the central index at $|z|=r$ by $\nu(r)$ for the power series in (1.8). We also regard $C, C_{1}$, and $C_{2}$ as positive constants, although their appearances at each time may not be the same number.

\section{A theorem about the function $f$}

THEOREM 2.1. Let $f$ be as in (1.8). Then there exists a sequence of $r$ tending to 1 such that

$$
\begin{gathered}
M(r ; \phi-\delta, \phi+\delta) \geq \frac{1}{\sqrt{3}}\left(M_{2}(r)+o(1)\right), \\
M_{2}(r ; \phi-\delta, \phi+\delta) \geq \frac{1}{\sqrt{3}}\left(M_{2}(r)+o(1)\right),
\end{gathered}
$$

for any $\theta_{0} \in(0,2 \pi]$ and for any $\delta \geq(6 \pi / \alpha)(\nu(r))^{-(1-\tau)(\alpha-1)}$, where $\tau$ is a real number with $1-\beta /(2 \alpha \beta+4)<\tau<1$.

Proof. The proof of Theorem 2.1 deeply depends on the proof of [5, Theorem 1]. Therefore, we will directly use some equations and inequalities from [5]. Consider

$$
f(z)=G(z)+H(z), \quad \text { where } G(z)=\sum_{k=0}^{J-1} a_{k} z^{k^{\alpha}}
$$

for $J=\left[v(r)^{1-\tau}\right]$. For any $\delta$ with $\delta \geq(6 \pi / \alpha) v(r)^{-(1-\tau)(\alpha-1)}$, [5, Lemma 1] implies

$$
\int_{\phi-\delta}^{\phi+\delta}\left|H\left(r e^{i \theta}\right)\right|^{2} d \theta>\frac{\delta}{3 \pi} \int_{0}^{2 \pi}\left|H\left(r e^{i \theta}\right)\right|^{2} d \theta .
$$

It follows from the Minkowski inequality and the above inequality that

$$
\begin{aligned}
& \left(\int_{\phi-\delta}^{\phi+\delta}|f|^{2} d \theta\right)^{1 / 2} \\
& \quad \geq\left(\int_{\phi-\delta}^{\phi+\delta}|H|^{2} d \theta\right)^{1 / 2}-\left(\int_{\phi-\delta}^{\phi+\delta}|G|^{2} d \theta\right)^{1 / 2} \\
& \quad \geq\left(\frac{\delta}{3 \pi} \int_{0}^{2 \pi}|H|^{2} d \theta\right)^{1 / 2}-\left(\int_{\phi-\delta}^{\phi+\delta}|G|^{2} d \theta\right)^{1 / 2} \\
& \quad \geq\left(\frac{\delta}{3 \pi}\right)^{1 / 2}\left(\left(\int_{0}^{2 \pi}|f|^{2} d \theta\right)^{1 / 2}-\left(\int_{0}^{2 \pi}|G(\theta)|^{2} d \theta\right)^{1 / 2}\right)-\left(\int_{\phi-\delta}^{\phi+\delta}|G|^{2} d \theta\right)^{1 / 2} .
\end{aligned}
$$


On the other hand, [5, equation (5)] gives, for $z=r e^{i \theta}$,

$$
|G(z)| \leq \frac{C}{-\alpha \log r}(\nu(r))^{(1-\tau)(\alpha \beta+2)}=: W(r) .
$$

Thus, we obtain from (2.4) and (2.5) that there is an $r_{1}$ such that for all $r$ with $r_{1}<$ $r<1$,

$$
\begin{aligned}
& M(r ; \phi-\delta, \phi+\delta)(2 \delta)^{1 / 2} \\
& \quad \geq\left(\frac{2 \delta}{3}\right)^{1 / 2}\left(M_{2}(r)+\frac{C}{-\alpha \log r} W(r)\right)-\frac{C}{-\alpha \log r} W(r) \delta^{1 / 2} .
\end{aligned}
$$

Similarly,

$$
\begin{aligned}
& M_{2}(r ; \phi-\delta, \phi+\delta)(2 \delta)^{1 / 2} \\
& \quad \geq\left(\frac{2 \delta}{3}\right)^{1 / 2}\left(M_{2}(r)+\frac{C}{-\alpha \log r} W(r)\right)-\frac{C}{-\alpha \log r} W(r) \delta^{1 / 2} .
\end{aligned}
$$

As shown in [5], we know that there exists a sequence of $r$ approaching one such that

$$
\mu(r) \geq(1-r)^{-(1+\beta / 2)}, \quad v(r) \leq C(\mu(r))^{1 /(1+\beta / 2)} .
$$

It follows that the first inequality in Theorem 2.1 is a consequence of (2.6) and (2.8). Similarly, we can prove the second inequality by using (2.7) and (2.8). Thus Theorem 2.1 is proved.

3. Results on the function $g$. Now we study the function $g$ in (1.11) and we assume that

$$
\gamma=\alpha(1+\beta)>2, \quad b_{k}=k^{\alpha}-(k-1)^{\alpha} .
$$

Thus, $b_{k}$ is strictly increasing and $b_{k} / b_{k+1}$ tends to 1 as $k$ goes to infinity. Let

$$
A_{k}=\left(\frac{k-1}{k}\right)^{\gamma / b_{k}}=\left(1-\frac{1}{k}\right)^{\gamma / b_{k}}, \quad I_{k}=\left[A_{k}, A_{k+1}\right) .
$$

Clearly, the sequence $A_{k}$ is strictly increasing to 1 when $k$ tends to infinity. Therefore, $I_{p} \cap I_{q}=\varnothing$ for $p \neq q$, and $[0,1)=\cup_{j=1}^{\infty} I_{j}$. Moreover, let $j$ be fixed, then, for $r \in I_{j}$ and $k \leq j-1$,

$$
\frac{(k+1)^{\gamma} r^{(k+1)^{\alpha}}}{k^{\gamma} r^{k^{\alpha}}}=\left(\frac{r}{A_{k+1}}\right)^{b_{k+1}} \geq\left(\frac{A_{j}}{A_{k+1}}\right)^{b_{k+1}} \geq 1 ;
$$

and for $r \in I_{j}$ and $k \geq j$,

$$
\frac{(k+1)^{\gamma} r^{(k+1)^{\alpha}}}{k^{\gamma} r^{k^{\alpha}}}=\left(\frac{r}{A_{k+1}}\right)^{b_{k+1}} \leq\left(\frac{A_{j+1}}{A_{k+1}}\right)^{b_{k+1}} \leq 1 .
$$


It turns out that the maximum term of $g$ on $|z|=r$, where $r \in I_{j}$, is

$$
\mu(r)=j^{\gamma} r^{j^{\alpha}} .
$$

THEOREM 3.1. If $g$ is defined as in (1.11), then

$$
\lim _{r \rightarrow 1^{-}} \frac{\log \mu(r)}{-\log (1-r)}=\frac{1}{1+\beta} .
$$

Proof. Let $j$ be fixed, then for any $r \in I_{j}=\left[A_{j}, A_{j+1}\right)$, we have

$$
\log r \leq \log A_{j+1}=\frac{\gamma}{(j+1)^{\alpha}-j^{\alpha}} \log \left(1-\frac{1}{j+1}\right) \leq-\frac{C}{j^{\alpha}} .
$$

Hence, noting $\log (1 / r) \sim 1-r$, we obtain, for $r \in I_{j}$, and $j$ is big,

$$
j^{\alpha} \geq-\frac{C}{\log r} \geq \frac{C}{1-r} .
$$

Consequently, for $r \in I_{j}$,

$$
\mu(r)=j^{\gamma} r^{j^{\alpha}} \geq A_{j}^{j^{\alpha}}\left(\frac{C}{1-r}\right)^{1+\beta}
$$

On the other hand, since $r \in I_{j}$, so

$$
\log r \geq \log A_{j}=\frac{\gamma}{j^{\alpha}-(j-1)^{\alpha}} \log \left(1-\frac{1}{j}\right) \geq-\frac{C}{j^{\alpha}} .
$$

Hence

$$
\mu(r) \leq j^{\gamma} \leq\left(\frac{C}{1-r}\right)^{1+\beta}
$$

Combining this with (3.9) and using $\lim _{j \rightarrow \infty} A_{j}^{j^{\alpha}}=e^{-(1+\beta)}$, we can find two positive constants $C_{1}$ and $C_{2}$ such that

$$
\frac{C_{1}}{(1-r)^{1+\beta}} \leq \mu(r) \leq \frac{C_{2}}{(1-r)^{1+\beta}},
$$

for all $r$ near to 1 . Thus the theorem is proved.

THEOREM 3.2. Let $g$ be as in (1.11). Then

$$
\lim _{r \rightarrow 1^{-}} \frac{\log M(r)}{\log \mu(r)}=1+\frac{1}{\alpha(1+\beta)} .
$$

Proof. Let $j$ be fixed, then, for $r \in I_{j}$,

$$
\begin{aligned}
M(r) & =f(r) \\
& =\mu(r)\left(\sum_{k=1}^{j-1}\left(\frac{k}{j}\right)^{\gamma} r^{k^{\alpha}-j^{\alpha}}+1+\sum_{k=j+1}^{\infty}\left(\frac{k}{j}\right)^{\gamma} r^{k^{\alpha}-j^{\alpha}}\right) \\
& =: \mu(r)\left(J_{1}+1+J_{2}\right) .
\end{aligned}
$$

In order to prove the theorem, we need to find upper and lower bounds of $J_{1}$ and $J_{2}$. 
For a fixed $j$, we have, for $r \in I_{j}$ and $k \leq j-1$,

$$
\left(\frac{k}{j}\right)^{\gamma} r^{k^{\alpha}-j^{\alpha}} \leq\left(\frac{k}{j}\right)^{\gamma} A_{j}^{-j^{\alpha}}
$$

Consequently, there is a positive constant $C$ such that the inequality

$$
J_{1}+1=\sum_{k=1}^{j}\left(\frac{k}{j}\right)^{\gamma} r^{k^{\alpha}-j^{\alpha}} \leq A_{j}^{-j^{\alpha}} \sum_{k=1}^{j}\left(\frac{k}{j}\right)^{\gamma} \leq C j
$$

holds for all large $j$ since $\sum_{k=1}^{j} k^{\gamma} \sim j^{\gamma+1} /(\gamma+1)$ for $\gamma>-1$, and $A_{j}^{-j^{\alpha}} \sim e^{1+\beta}$.

Now to find a lower bound of $J_{1}$. Indeed, for $r \in I_{j}$, there is a positive constant $C$ such that

$$
J_{1}+1=\sum_{k=1}^{j}\left(\frac{k}{j}\right)^{\gamma} r^{k^{\alpha}-j^{\alpha}} \geq \sum_{k=1}^{j}\left(\frac{k}{j}\right)^{\gamma} \geq C j,
$$

for all large $j$.

Now to estimate $J_{2}$. For $r \in I_{j}$, and $k \geq j+1$,

$$
\begin{aligned}
\left(\frac{k}{j}\right)^{\gamma} r^{k^{\alpha}-j^{\alpha}} & \leq\left(\frac{k}{j}\right)^{\gamma} A_{j+1}^{k^{\alpha}-j^{\alpha}}=\left(\frac{k}{j}\right)^{\gamma} A_{j+1}^{k^{\alpha}} A_{j+1}^{-j^{\alpha}} \\
& =\left(\frac{k}{j}\right)^{\gamma} \exp \left\{\frac{\gamma k^{\alpha}}{(j+1)^{\alpha}-j^{\alpha}} \log \left(1-\frac{1}{j+1}\right)\right\} A_{j+1}^{-j^{\alpha}} \\
& \leq\left(\frac{k}{j}\right)^{\gamma} \exp \left\{\frac{\gamma k^{\alpha}}{(j+1)^{\alpha}-j^{\alpha}}\left(-\frac{1}{j+1}\right)\right\} A_{j+1}^{-j^{\alpha}} .
\end{aligned}
$$

Thus, for all large $j$ and for $r \in I_{j}$,

$$
\begin{aligned}
J_{2} & =\sum_{k=j+1}^{\infty}\left(\frac{k}{j}\right)^{\gamma} r^{k^{\alpha}-j \alpha} \\
& \leq A_{j+1}^{-j^{\alpha}} \sum_{k=j+1}^{\infty}\left(\frac{k}{j}\right)^{\gamma} \exp \left\{\frac{\gamma k^{\alpha}}{(j+1)^{\alpha}-j^{\alpha}}\left(-\frac{1}{j+1}\right)\right\} \\
& \leq A_{j+1}^{-j^{\alpha}} \sum_{k=j+1}^{\infty}\left(\frac{k}{j}\right)^{\gamma} \exp \left\{-\gamma\left(\frac{k}{j}\right)^{\alpha} 2^{-(\alpha+1)}\right\} \\
& \leq A_{j+1}^{-j^{\alpha}} j \int_{1}^{\infty} x^{\gamma} \exp \left\{-\gamma x^{\alpha} 2^{-(\alpha+1)}\right\} d x \\
& \leq C j .
\end{aligned}
$$

It follows from (3.14), (3.16), (3.17), and (3.19) that there are two positive constants $C_{1}$ and $C_{2}$ such that

$$
C_{1} \mu(r) j \leq M(r) \leq C_{2} \mu(r) j,
$$

for any $r \in I_{j}$, and all large $j$. Moreover, as $r \in I_{j}$,

$$
\mu(r) \leq j^{\gamma}=\mu(r) r^{-j^{\alpha}} \leq C_{2} \mu(r) .
$$


Therefore,

$$
C_{1}(\mu(r))^{1+1 / \gamma} \leq M(r) \leq C_{2}(\mu(r))^{1+1 / \gamma},
$$

which implies the theorem.

COROLLARY 3.3. Let $g$ be as in (1.11). Then

$$
\lim _{r \rightarrow 1^{-}} \frac{\mu(r)}{M(r)}=0
$$

Obviously, the corollary is a straightforward consequence of Theorem 3.2.

THEOREM 3.4. Let $g$ be as in (1.11). Then

$$
\lim _{r \rightarrow 1^{-}} \frac{L(r)}{M(r)}=0
$$

Proof. For any $\epsilon>0$, we have from the above corollary that there exists $r_{0}$ such that, for $r \geq r_{0}$,

$$
\mu(r) \leq \epsilon M(r)
$$

Thus, for all $r$ near to 1 ,

$$
\begin{aligned}
M^{2}(r) & =f^{2}(r)=\left(\sum_{k=1}^{\infty} k^{\gamma} r^{k^{\alpha}}\right) f(r)=\sum_{k=1}^{\infty} k^{2 \gamma} r^{2 k^{\alpha}}+\sum_{k=1}^{\infty} k^{\gamma} r^{k^{\alpha}}\left(f(r)-k^{\gamma} r^{k^{\alpha}}\right) \\
& \geq M_{2}(r)+\sum_{k=1}^{\infty} k^{\gamma} r^{k^{\alpha}}(f(r)-\mu(r))=M_{2}(r)+M(r)(M(r)-\mu(r)) \\
& \geq L^{2}(r)+(1-\epsilon) M(r) .
\end{aligned}
$$

Therefore, for all $r$ near to 1 ,

$$
L(r) \leq \sqrt{\epsilon} M(r)
$$

It follows that the theorem is proved.

THEOREM 3.5. If $g$ is defined as in (1.11), then

$$
\limsup _{r \rightarrow 1^{-}} \frac{\log L(r)}{\log M(r)}=1 \text {. }
$$

Proof. By a little computation, we know that $|g(-r)|=L(r)$. Moreover, noting that $k^{\alpha}$ is odd or even if $k$ is odd or even, we have

$$
L(r)=\left|-\sum_{k=1}^{\infty}(2 k-1)^{\gamma} r^{(2 k-1)^{\alpha}}+\sum_{k=1}^{\infty}(2 k)^{\gamma} r^{(2 k)^{\alpha}}\right|
$$

Set

$$
h(r)=\sum_{k=1}^{\infty}(2 k)^{\gamma} r^{(2 k)^{\alpha}}
$$


Then

$$
h(r)=2^{\gamma} g\left(r^{2^{\alpha}}\right)=2^{\gamma} M\left(r^{2^{\alpha}}\right) .
$$

Therefore

$$
L(r)=|h(r)-g(r)+h(r)|=\left|2^{\gamma+1} M\left(r^{2^{\alpha}}\right)-M(r)\right| .
$$

On the other hand, we have from (3.12) and (3.22) that there is a positive constant $C$ and a sequence $r_{n}$, which goes to 1 , such that

$$
M\left(r_{n}\right) \sim C\left(1-r_{n}\right)^{s} \text { for } n=1,2, \ldots,
$$

where $s=-(1+\beta+1 / \alpha)$. Thus

$$
\begin{aligned}
\lim _{n \rightarrow \infty} & \frac{\log \left|2^{\gamma+1} M\left(r_{n}^{2^{\alpha}}\right) M^{-1}\left(r_{n}\right)-1\right|}{\log M\left(r_{n}\right)} \\
& =\lim _{n \rightarrow \infty} \frac{\log \left|2^{\gamma+1}\left(1-r_{n}^{2^{\alpha}}\right)^{s}\left(1-r_{n}\right)^{s}-1\right|}{-s \log \left(1-r_{n}\right)+O(1)} \\
& =\lim _{n \rightarrow \infty} \frac{2^{\gamma+1} s\left(1-r_{n}^{2^{\alpha}}\right)^{s-1} 2^{\alpha} r_{n}^{2^{\alpha}-1}\left(1-r_{n}\right)^{-s+1}+2^{\gamma+1}\left(1-r_{n}^{2^{\alpha}}\right)^{s} s\left(1-r_{n}\right)^{-s}}{s 2^{\gamma+1}\left(1-r_{n}^{2 \alpha}\right)^{s}\left(1-r_{n}\right)^{-s}-1} \\
& =0 .
\end{aligned}
$$

It follows that

$$
\limsup _{r \rightarrow 1^{-}} \frac{\log L(r)}{\log M(r)}=1+\limsup _{r \rightarrow 1^{-}} \frac{\log \left|2^{\gamma+1} M\left(r^{2^{\alpha}}\right) M^{-1}(r)-1\right|}{\log M(r)} \geq 1 .
$$

Thus the theorem is proved.

COROLlary 3.6. If $g$ is defined as in (1.11) and $a$ is a complex number, then the Nevanlinna deficiency of $f$ at $a$ is zero.

Proof. Applying Jensen's formula to $g-a$ gives

$$
N(g, r, a)=\frac{1}{2 \pi} \int_{0}^{2 \pi} \log \left|g\left(r e^{i \theta}\right)-a\right| d \theta+O(1) \geq \log |L(r)-| a||+O(1),
$$

where the last inequality holds on a sequence of $r_{n}$, on which $L(r)$ goes to infinity as $r_{n}$ tends to one. Theorem 3.5 ensures the existence of such a sequence. Thus we have

$$
\begin{aligned}
\delta(g, a) & =1-\limsup _{r \rightarrow 1^{-}} \frac{N(g, r, a)}{T(g, r)} \\
& \leq 1-\limsup _{r \rightarrow 1^{-}} \frac{N(f, r, a)}{\log M(r)} \\
& \leq 1-\limsup _{r \rightarrow 1^{-}} \frac{\log L(r)}{\log M(r)}=0 .
\end{aligned}
$$

It follows that the corollary is proved. 
REMARK 3.7. The corollary implies that the function $g$ in (1.11) takes every complex value infinitely many times in the unit disc.

THEOREM 3.8. If $g$ is defined as in (1.11), then

$$
\lim _{r \rightarrow 1^{-}} \frac{\log M_{2}(r)}{\log \mu(r)}=1+\frac{1}{2 \gamma} .
$$

Proof. For a fixed $j$, we have, for $r \in I_{j}$,

$$
\begin{aligned}
M_{2}^{2}(r) & =\sum_{k=1}^{\infty} k^{2 \gamma} r^{2 k^{\alpha}} \\
& =\mu^{2}(r)\left(\sum_{k=1}^{j}\left(\frac{k}{j}\right)^{2 \gamma} r^{2 k^{\alpha}-2 j^{\alpha}}+\sum_{k=j+1}^{\infty}\left(\frac{k}{j}\right)^{2 \gamma} r^{2 k^{\alpha}-2 j^{\alpha}}\right) \\
& =: \mu^{2}(r)\left(P_{1}+P_{2}\right) .
\end{aligned}
$$

We now estimate $P_{1}$ and $P_{2}$. For $r \in I_{j}$ and $k \leq j-1$,

$$
\begin{aligned}
& P_{1}=\sum_{k=1}^{j}\left(\frac{k}{j}\right)^{2 \gamma} r^{2 k^{\alpha}-2 j^{\alpha}} \leq A_{j}^{-2 j^{\alpha}} \sum_{k=1}^{j}\left(\frac{k}{j}\right)^{2 \gamma} \leq C j, \\
& P_{1}=\sum_{k=1}^{j}\left(\frac{k}{j}\right)^{2 \gamma} r^{2 k^{\alpha}-2 j^{\alpha}} \geq \sum_{k=1}^{j}\left(\frac{k}{j}\right)^{2 \gamma} \geq C j .
\end{aligned}
$$

Moreover,

$$
P_{2}=\sum_{k=j+1}^{\infty}\left(\frac{k}{j}\right)^{2 \gamma} r^{2 k^{\alpha}-j^{2 \alpha}} \leq A_{j+1}^{-2 j^{\alpha}} j \int_{1}^{\infty} x^{\gamma} \exp \left\{-\gamma x^{\alpha} 2^{-(\alpha+2)}\right\} d x \leq C j .
$$

It follows that there exist two positive constants $C_{1}$ and $C_{2}$ such that

$$
C_{1} \mu^{2}(r) j \leq M_{2}^{2}(r) \leq C_{2} \mu^{2}(r) j,
$$

for all large $j$ and $r \in I_{j}$. It turns out from (3.21) that the theorem is proved.

COROLlary 3.9. If $g$ is defined as in (1.11), then

$$
\lim _{r \rightarrow \infty} \frac{\log M_{2}(r)}{\log M(r)}=\frac{1+2 \gamma}{2+2 \gamma} .
$$

The proof of the corollary follows from Theorems 3.2 and 3.8.

The results of the following theorem and corollary are better than those of Theorem 2.1. Moreover, since we are dealing with the functions in (1.11), we can give a direct proof without using anything from [5].

THEOREM 3.10. Let $g$ be as in (1.11). Then for any $\theta_{0} \in(0,2 \pi]$ and any $r>0$,

$$
\left(\frac{1}{2 T} \int_{\theta_{0}-T}^{\theta_{0}+T}\left|g\left(r e^{i \theta}\right)\right|^{2} d \theta\right)^{1 / 2} \geq\left(\frac{1}{\sqrt{3}}+o(1)\right) M_{2}(r)
$$

for any $T$ with $\pi \geq T \geq(1-r)^{s}$ for $r$ near to 1 , where $s=(4 \gamma+1)(2 \alpha-3) / 4 \alpha(\gamma+2)$. 
Proof. For any $r \in I_{j}$, and $\theta_{0} \in(0,2 \pi]$, we write

$$
\begin{aligned}
F(\theta) & =g\left(r e^{\left(\theta_{0}-\theta\right) i}\right)=\sum_{k=1}^{\infty} k^{\gamma} r^{k^{\alpha}} e^{\theta_{0} k^{\alpha} i} e^{-k^{\alpha} \theta i}=\sum_{k=1}^{\infty} a_{k} e^{-k^{\alpha} \theta i} \\
& =\sum_{k=1}^{\left[j^{t}\right]} a_{k} e^{-k^{\alpha} \theta i}+\sum_{k=\left[j^{t}\right]+1}^{\infty} a_{k} e^{-k^{\alpha} \theta i}=G(\theta)+H(\theta),
\end{aligned}
$$

where $t$ is a positive real number with $t \leq(\gamma+1 / 2) /(\gamma+2)$. Let $d=\left[j^{t}\right]+1$, then

$$
\begin{aligned}
2 T^{2} \int_{-T}^{T}|H(\theta)|^{2} d \theta & \geq \int_{-T}^{T}|H(\theta)|^{2}\left(T^{2}-\theta^{2}\right) d \theta \\
& =\sum_{p, q \geq d} \int_{-T}^{T} a_{p} \overline{a_{q}} e^{-\theta\left(p^{\alpha}-q^{\alpha}\right) i}\left(T^{2}-\theta^{2}\right) d \theta \\
& =\sum_{k=d}^{\infty}\left|a_{k}\right|^{2} \frac{4 T^{3}}{3}+\sum_{p, q \geq d, p \neq q} a_{p} \overline{a_{q}} \frac{4 \sin \left(p^{\alpha}-q^{\alpha}\right) T-\left(p^{\alpha}-q^{\alpha}\right) T \cos \left(p^{\alpha}-q^{\alpha}\right) T}{\left(p^{\alpha}-q^{\alpha}\right)^{3}} \\
& \geq \sum_{k=d}^{\infty}\left|a_{k}\right|^{2} \frac{4 T^{3}}{3}-\sum_{p, q \geq d, p \neq q}\left(\left|a_{p}\right|^{2}+\left|a_{q}\right|^{2}\right) \frac{4 T}{\left(p^{\alpha}-q^{\alpha}\right)^{2}} \\
& =\sum_{k=d}^{\infty}\left|a_{k}\right|^{2} \frac{4 T^{3}}{3}-2 \sum_{p, q \geq d, p \neq q}\left|a_{q}\right|^{2} \frac{4 T}{\left(p^{\alpha}-q^{\alpha}\right)^{2}} \\
& =\sum_{k=d}^{\infty}\left|a_{k}\right|^{2} \frac{4 T^{3}}{3}-2 \sum_{q=d}^{\infty} \sum_{p=d ; p \neq q}^{\infty}\left|a_{q}\right|^{2} \frac{4 T}{p^{2} d^{2 \alpha-2} \alpha} \\
& =\left(\frac{4 T^{3}}{3}-\frac{4 T}{d^{2 \alpha-2} \alpha} \sum_{p=1}^{\infty} \frac{1}{p^{2}}\right) \sum_{k=d}^{\infty}\left|a_{k}\right|^{2} \\
& =: B_{d} \sum_{k=d}^{\infty}\left|a_{k}\right|^{2} \\
= & B_{d} \frac{1}{2 \pi} \int_{0}^{2 \pi} \frac{\mid H(\theta)}{\mid d \theta .}
\end{aligned}
$$

It follows from the Minkowski inequality and (3.46) that

$$
\begin{aligned}
\left(\frac{1}{2 T} \int_{-T}^{T}|F(\theta)|^{2} d \theta\right)^{1 / 2} & \geq\left(\frac{1}{2 T} \int_{-T}^{T}|H(\theta)|^{2} d \theta\right)^{1 / 2}-\left(\frac{1}{2 T} \int_{-T}^{T}|G(\theta)|^{2} d \theta\right)^{1 / 2} \\
& \geq\left(\frac{B_{d}}{2 T^{2}} \frac{1}{2 T} \frac{1}{2 \pi} \int_{0}^{2 \pi}|H(\theta)|^{2} d \theta\right)^{1 / 2}-\left(\frac{1}{2 T} \int_{-T}^{T}|G(\theta)|^{2} d \theta\right)^{1 / 2} \\
\geq & \left(\frac{B_{d}}{8 T^{3} \pi}\right)^{1 / 2}\left(\left(\int_{0}^{2 \pi}|F(\theta)|^{2} d \theta\right)^{1 / 2}-\left(\int_{0}^{2 \pi}|G(\theta)|^{2} d \theta\right)^{1 / 2}\right) \\
& -\left(\frac{1}{2 T} \int_{-T}^{T}|G(\theta)|^{2} d \theta\right)^{1 / 2} .
\end{aligned}
$$


On the other hand, we have, for any $T \in(0, \pi]$ and $r \in I_{j}$,

$$
\left(\frac{1}{2 T} \int_{-T}^{T}|G(\theta)|^{2} d \theta\right)^{1 / 2} \leq \sum_{k=1}^{d-1} k^{\gamma} r^{k^{\alpha}} \leq \sum_{k=1}^{d-1} k^{\gamma} \leq C d^{\gamma+1} \leq C j^{(\gamma+1 / 2)(\gamma+1) /(\gamma+2)}
$$

However, for $r \in I_{j}$, we have from (3.42) and (3.9) that

$$
\left(\frac{1}{2 \pi} \int_{0}^{2 \pi}|F(\theta)|^{2} d \theta\right)^{1 / 2}=\left(\frac{1}{2 \pi} \int_{0}^{2 \pi}\left|g\left(r e^{i \theta}\right)\right|^{2} d \theta\right)^{1 / 2}=M_{2}(r) \geq C j^{\gamma+1 / 2} .
$$

It turns out from (3.48) and (3.49) that

$$
\left(\frac{1}{2 T} \int_{-T}^{T}|G(\theta)|^{2} d \theta\right)^{1 / 2}=o\left(M_{2}(r)\right)
$$

Thus we have from (3.47) and the definition of $B_{d}$ that

$$
\begin{aligned}
\left(\frac{1}{2 T} \int_{\theta_{0}-T}^{\theta_{0}+T}\left|g\left(r e^{i \theta}\right)\right|^{2} d \theta\right)^{1 / 2} & =\left(\frac{1}{2 T} \int_{-T}^{T}|F(\theta)|^{2} d \theta\right)^{1 / 2} \\
& \geq M_{2}(r)\left[\left(\frac{1}{3}-\frac{1}{2 \alpha T^{2} d^{2 \alpha-2}}\right)^{1 / 2}(1+o(1))-o(1)\right] \\
& \geq M_{2}(r)\left(\frac{1}{\sqrt{3}}-\frac{1}{\sqrt{2 \alpha} T d^{\alpha-1}}-o(1)\right) .
\end{aligned}
$$

It follows that the theorem holds when

$$
T \geq 1 / d^{\alpha-3 / 2} \geq(1-r)^{t(\alpha-(3 / 2)) / \alpha}
$$

Clearly, we have the following corollary.

COROLlary 3.11. If $g$ is defined as in (1.11), then there is a positive constant $C$ such that for any $\theta_{0} \in(0,2 \pi]$, and any $r$ near to 1 , we have

$$
\max _{\left|\theta-\theta_{0}\right| \leq T}\left|g\left(r e^{i \theta}\right)\right| \geq C M^{(1+2 \gamma) /(2 \gamma+2)}(r)
$$

for $T$ with $\pi \geq T \geq(1-r)^{s}$, where $s=(4 \gamma+1)(2 \alpha-3) / 4 \alpha(\gamma+2)$.

Proof. Clearly, for any $\theta_{0} \in(0,2 \pi]$, and any $r$ near to 1 , the theorem gives

$$
\max _{\left|\theta-\theta_{0}\right| \leq T}\left|g\left(r e^{i \theta}\right)\right| \geq\left(\frac{1}{\sqrt{\sqrt{3}}}+o(1)\right) M_{2}(r)
$$


for $T$ with $\pi \geq T \geq C(1-\gamma)^{s}$, where $s=(4 \gamma+1)(2 \alpha-3) / 4 \alpha(\gamma+2)$. Furthermore, we have from (3.17), (3.12), and (3.19) that

$$
M_{2}^{2}(r) \geq C \mu^{2}(r) j \geq C M(r) \mu(r) \geq C M^{(1+2 \gamma) /(1+\gamma)}(r)
$$

for all $r$ near to 1 . It follows that the corollary is proved.

\section{REFERENCES}

[1] P. Erdös and A. J. Macintyre, Integral functions with gap power series, Proc. Edinburgh Math. Soc. (2) 10 (1954), 62-70.

[2] C. N. Linden, The minimum modulus of functions of slow growth in the unit disk, Mathematical Essays Dedicated to A. J. Macintyre, Ohio University Press, Ohio, 1970, pp. 237-246.

[3] P. J. Nicholls and L. R. Sons, Minimum modulus and zeros of functions in the unit disc, Proc. London Math. Soc. (3) 31 (1975), no. 1, 99-113.

[4] L. R. Sons, Unbounded functions in the unit disc, Int. J. Math. Math. Sci. 6 (1983), no. 2, 201-242.

[5] _ On singular radii, Complex Variables Theory Appl. 12 (1989), no. 1-4, 71-75.

[6] A. Wiman, Über den Zusammenhang zwischen dem Maximalbetrage einer analytischen Funktion und dem grössten Betrage bei gegebenem Argumente der Funktion, Acta Math. 41 (1916), 1-28 (German).

L. R. SONS: Department of Mathematical Sciences, Northern IllinOIS University, DeKALB, IL 60115, USA

E-mail address: sons@math . niu.edu

Zhuan Ye: Department of Mathematical Sciences, Northern IllinOis University, DeKalb, IL 60115, USA

E-mail address: ye@math . niu.edu 


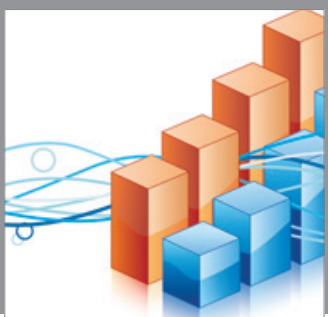

Advances in

Operations Research

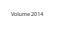

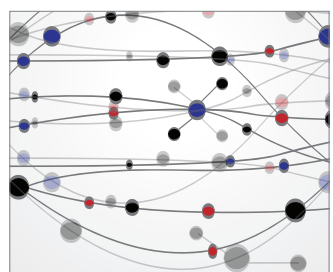

\section{The Scientific} World Journal
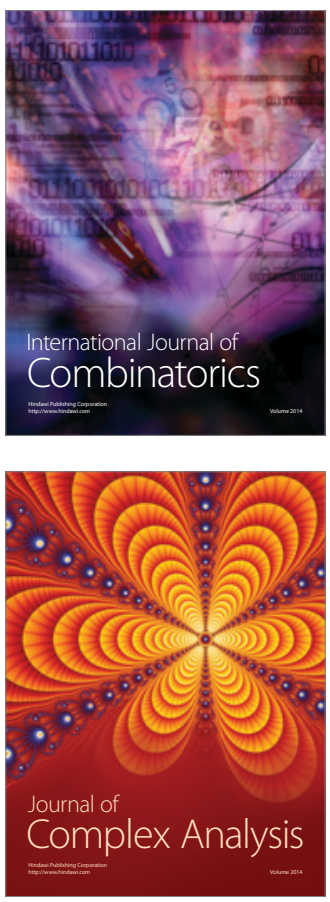

International Journal of

Mathematics and

Mathematical

Sciences
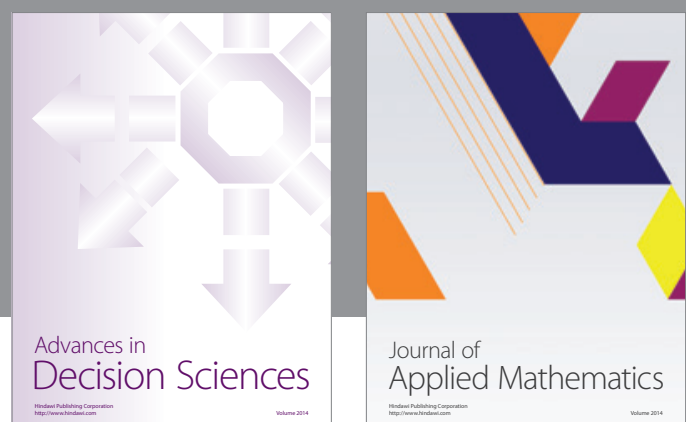

Journal of

Applied Mathematics
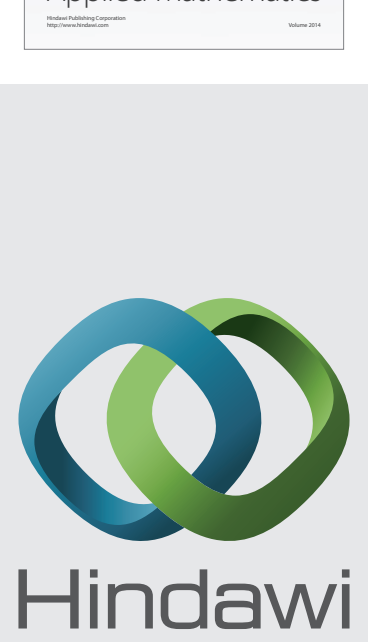

Submit your manuscripts at http://www.hindawi.com
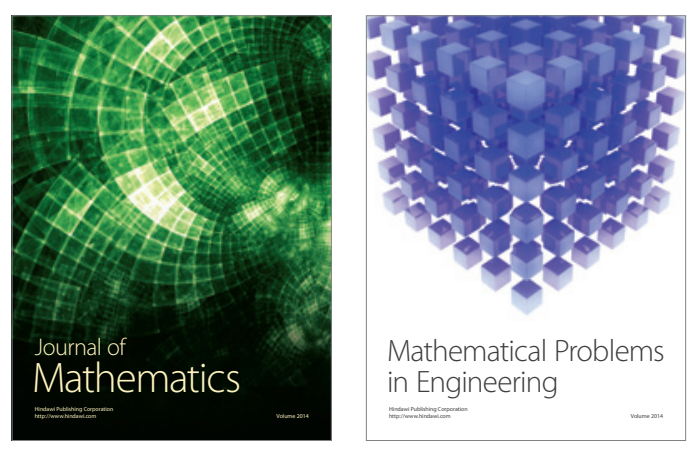

Mathematical Problems in Engineering
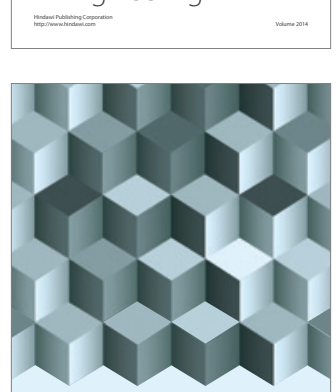

Journal of

Function Spaces
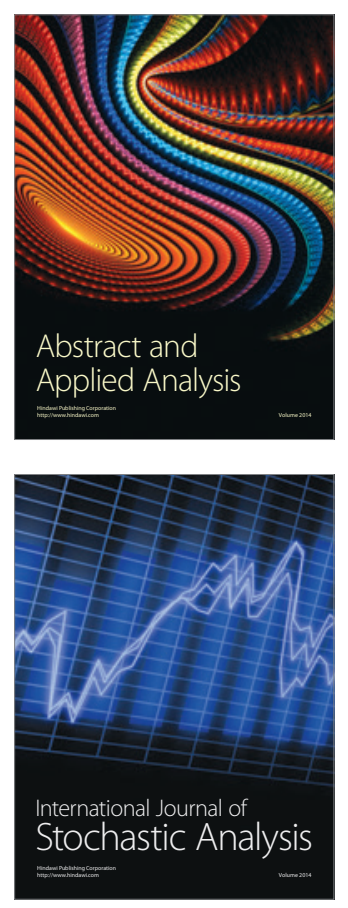

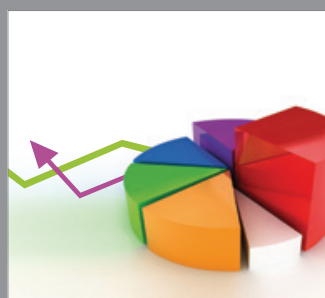

ournal of

Probability and Statistics

Promensencen
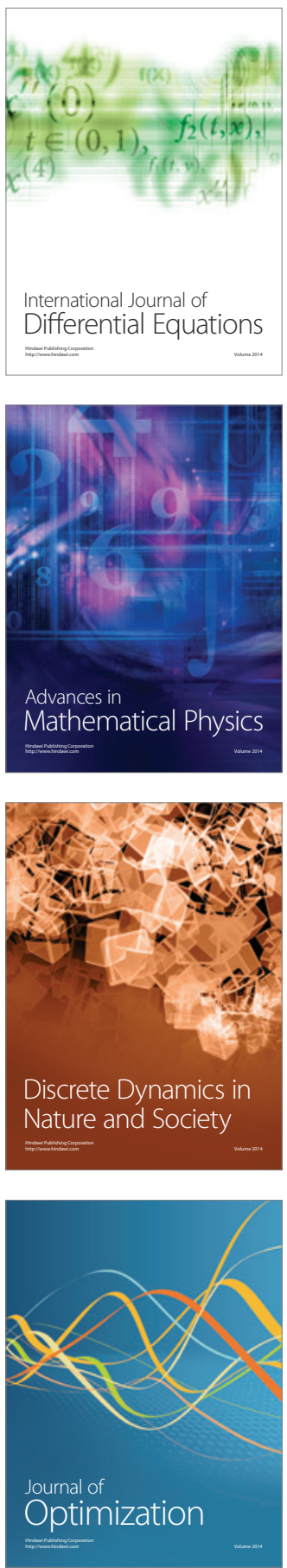